\title{
Datos prelimirares de uso de clases experimentales en la enseñanza de las ciencias
}

DOI: $10.46814 / \operatorname{lajdv3n3-030}$

Recebimento dos originais: 01/052021

Aceitação para publicação: 31/06/2021

\section{Doutorando João Soares Santos}

Doutorando em Ciencias da Educação, pela Universidade Nacional de Rosario (UNR Argentina), mestre em Biotecnologia, Licenciado em Ciencias Biologicas e Pedagogia.

E-mail: joao.soares.2@hotmail.com

\section{Profa. Dra Érika Cristina Teixeira Dos Anjos}

Doutora em Ciencias Biologicas pela Universidade Federal de Pernambuco (UFPE)

E-mail: erikaanjos@yahoo.com.br

\section{Prof. Dr Fernando Carlos Avendaño}

Doctor en Humanidades y Artes (mención Ciencias de la Educación) pela Universidad Nacional De Rosario (UNR).

E-mail: fernandoavendano90@gmail.com

\section{RESUMEN}

O presente trabajo tem a finalidade de Analizar la Importancia del uso de las Metodologías activas en la Construcción del Conocimiento de Ciencias de la Educación Fundamental bajo la Perspectiva del Desarrollo de Competencias y Habilidades. Por lo tanto la Enseñanza de las Ciencias es muy importante, ya que aborda los principios científicos generales y, también, aplicaciones tecnológicas. Los conceptos y teorías científicas no tienen valores en sí mismos, como sistemas abstractos de pensamiento, sino como instrumentos que nos ayudan a entender el mundo en el que vivimos con el fin de orientar nuestras acciones a nivel individual y social. La práctica vinculada a la teoría hace mucha diferencia para una clase contextualizada, donde los estudiantes pueden ver la importancia de los contenidos abordados en la enseñanza de las Ciencias, además de ofrecer a los mismos una mayor claridad para que puedan realmente interpretar o sea hacer parte del estudio en cuestión. . Es visible hoy en día la necesidad de la experimentación durante las clases como instrumento de enseñanza, porque el estímulo y el interés de los estudiantes pasa a ser mucho mayor, ya que los mismos pueden visualizar el contenido de forma diferente, o sea, pasan a analizar ciertas cuestiones como si hicieran parte de ella.

Por lo tanto, la práctica experimental tiene un papel más amplio de lo que se espera, porque desarrolla en los alumnos un mayor interés, además de despertar habilidades que no aparecía en las clases teóricas, por ejemplo.

Palabras Clave: aprendizaje, currículum, formación de profesores, clases prácticas, metodologías alternativas, metodologías tradicionales y enseñanza de ciencias.

\section{INTRODUCCIÓN}

La Enseñanza de las Ciencias es muy importante, ya que aborda los principios científicos generales y, también, aplicaciones tecnológicas. Los conceptos y teorías científicas no tienen valores en sí mismos, como sistemas abstractos de pensamiento, sino como instrumentos que nos ayudan a entender el mundo en el que vivimos con el fin de orientar nuestras acciones a nivel individual y social. 
Es en la época de la escuela que los estudiantes aprenden a gustar o no de la materia de Ciencias, ya que la misma posibilita la comprensión de los acontecimientos pasados, o sea, permite entender los procesos que rigen nuestra vida en la Tierra. Es posible comprobar la expectativa de los educandos durante la semana para tener clases de Ciencias, porque siempre le preguntan lo que será el pasado de diferente en el aula.

Através de toda la dificultad de los alumnos frente a esta materia fue posible informar sobre la importancia de las clases prácticas experimentales para enseñar Ciencias en la educación básica.Después de mucha investigación, se puede entender que la experimentación durante las clases, no sólo está en despertar el interés por la Ciencia en los alumnos, sino de hacer más comprensible los contenidos abordados en la teoría.

La práctica vinculada a la teoría hace mucha diferencia para una clase contextualizada, donde los estudiantes pueden ver la importancia de los contenidos abordados en la enseñanza de las Ciencias, además de ofrecer a los mismos una mayor claridad para que puedan realmente interpretar o sea hacer parte del estudio en cuestión.

Es visible hoy en día la necesidad de la experimentación durante las clases como instrumento de enseñanza, porque el estímulo y el interés de los estudiantes pasa a ser mucho mayor, ya que los mismos pueden visualizar el contenido de forma diferente, o sea, pasan a analizar ciertas cuestiones como si hicieran parte de ella.

Por lo tanto, la práctica experimental tiene un papel más amplio de lo que se espera, porque desarrolla en los alumnos un mayor interés, además de despertar habilidades que no aparecía en las clases teóricas, por ejemplo.

\section{FUNDAMENTACIÓN:}

\subsection{LA ENSEÑANZA DE LAS CIENCIAS CON ENFOQUE EN LA ENSEÑANZA} FUNDAMENTAL.

Las divergencias sobre la naturaleza de la ciencia ofrecen diferentes interpretaciones y estilos de conducir a las actividades experimentales. Mucho se ha discutido acerca de la relevancia de las actividades experimentales integradas en el contexto escolar, ya sean hechas por el profesor o por los alumnos. El modo y los medios como son aplicadas, así como el papel que desarrollan en el aula. Hechos estos que nos despertaron a las implicaciones que esto conlleva en cuanto a las concepciones del laboratorio didáctico, en especial de las Ciencias.

Ante tal contexto, realizamos un relevamiento de artículos, disertaciones y tesis sobre actividades experimentales como estrategia de enseñanza de las Ciencias, dirigida a la enseñanza 
fundamental, buscando, así, apoyar a las investigaciones en los que estamos involucrados en nuestro trabajo de investigación.

Constatamos que las discusiones referentes al uso de actividades experimentales en la enseñanza de las Ciencias se intensificaron a partir de la segunda mitad del siglo XX, con el surgimiento de nuevos modelos para el desarrollo científico. Epistemólogos como Thomas Kuhn, Karl Popper, Gaston Bachelard, entre otros, al cuestionarse la naturaleza del conocimiento humano, desde la perspectiva de la interacción no neutral entre el sujeto y el objeto, han contribuido a una maduración sobre la función histórica del experimento, al permitir una mejor comprensión de la relación entre teoría - experimento - realidad, por lo que es posible entender las visiones de la ciencia incorporadas en las diversas propuestas de enseñanza experimental.

En esa época, mientras que los filósofos y epistemólogos seguían sus polémicas acerca de la ciencia, los científicos desarrollaron proyectos como el Physical Science StudyCommitee (PSSC) y el Harvard Project Physics, en los Estados Unidos, Nuffield, en Inglaterra, y el Proyecto de Enseñanza de la Física (PEF), en Brasil, que tenían como idea básica de reproducir la práctica del científico profesional, llevando a los estudiantes a "hacer ciencia", la ciencia del científico. La inserción de estos proyectos, orientados a la práctica de laboratorios, guiados por una visión rígida y tradicional del método científico, tenía por objeto proporcionar los medios más esperanzadora y eficaces para las demostraciones y las comprobaciones hasta entonces solo en los libros de texto o en las explicaciones del profesor.

A partir de la década de 1970, la teoría de la enseñanza y el aprendizaje que imperaba, la instrucción programada, propuesta por Skinner, aún identificada en apuntes y libros de texto, acabó siendo cuestionada. La "competencia" fue realzada, nuevas teorías de aprendizaje, denominadas constructivistas, apuntaron para la valoración de los procesos mentales y el lema de "aprender a aprender" destacó, proporcionando cambios en el ámbito social, político y cultural.

En consecuencia, surgieron nuevas propuestas de uso del laboratorio para la enseñanza de las ciencias, que se centran en aspectos más relacionados al análisis de las explicaciones e ideas previas de los alumnos.

Entendemos que las innovaciones ocurridas han contribuido a una multiplicidad de tendencias y modalidades, según explicitaremos en nuestra revisión bibliográfica descrita a continuación, proporcionando un análisis más cautelosa respecto a la relevancia de las actividades experimentales en el contexto del aula y de los obstáculos para la aplicación de dichas estrategias.

La función de la enseñanza experimental está relacionada con la conciencia de la necesidad de adopción, por el profesor, de una postura diferente sobre cómo enseñar y aprender ciencias. La actitud del profesor debe basarse, según Hodson (1994), en la intención de ayudar a los alumnos en la 
exploración, desarrollo y modificación de sus "concepciones ingenuos' acerca de un fenómeno para concepciones científicas, sin desprecian las.

Aún con relación a la enseñanza de las Ciencias en la educación primaria, se puede destacar la dificultad del alumno para relacionar la teoría desarrollada en el aula con la realidad a su alrededor, y es por este motivo que las actividades prácticas experimentales son de suma importancia ya que proporcionan al alumno vivenciar la realidad discutida en el aula a través de teorías científicas (SERAFÍN, 2001).

La realización de experimentos en Ciencias, representa una excelente herramienta para que el alumno realice la experimentación del contenido y pueda establecer la dinámica y la indisociable relación entre la teoría y la práctica. La importancia de la experimentación en el proceso de aprendizaje también es discutida por Bazin (1987) que, en una experiencia de educación no formal de las Ciencias, la apuesta por la mayor importancia de esta metodología en relación a la simple memorización de la información, el método tradicionalmente empleado en las aulas.

La función de la enseñanza experimental está directamente relacionada con la conciencia de la necesidad de adopción, por el profesor, de una postura diferente sobre cómo enseñar y aprender ciencias. La actitud del profesor debe basarse, según Hodson (1994):

En la intención de ayudar a los alumnos en la exploración, desarrollo y modificación de sus "concepciones ingenuos' acerca de un fenómeno para concepciones científicas, sin desprecian las. Los estudiantes deben ser alentados a explorar sus opiniones, invitándoles a reflexionar sobre el potencial que sus ideas tienen para explicar fenómenos y apuntes recogidos en la actividad experimental.

Considerando que la teoría está hecha de conceptos que son abstracciones de la realidad (SERAFÍN, 2001), podemos inferir que el alumno que no reconoce el conocimiento científico en su vida cotidiana, y no fue capaz de entender la teoría. Segundo Freire (1997), para compreender a teoria é preciso experienciá-la.

La educación en Ciencias debe proporcionar a los estudiantes la oportunidad de desarrollar capacidades que despierten en ellos la inquietud ante lo desconocido, buscando explicaciones lógicas y razonables, llevando a los estudiantes a desarrollar posturas críticas, realizar juicios y tomar decisiones importantes (BIZZO, 1998).De este modo, las clases prácticas pueden ayudar en el desarrollo de conceptos científicos, además de permitir que los estudiantes aprendan cómo abordar objetivamente el mundo y resolver problemas complejos (LUNETTA, 1991). La experimentación se prioriza el contacto de los alumnos con los fenómenos químicos posibilitando al alumno la creación de los modelos que tengan sentidos para ellos a partir de sus propias observaciones (GIORDAN, 1999).

Con base en autores como Gaspar (2009), Krasilchik (2004) y Carvalho et. al., (2007), se puede afirmar que: 
Con la realización de juicios y no sólo con las clases magistrales, el alumno venga a reestructurar su pensamiento, que se inicia en la educación científica de forma más eficaz.

La importancia del trabajo práctico es incuestionable en la Ciencia y debería ocupar un lugar central en su enseñanza (SMITH, 1975).

Sin embargo, el aspecto formativo de las actividades prácticas experimentales se ha hecho muchas veces, al carácter superficial, mecánico y repetitivo en detrimentos de los aprendizajes teóricoprácticos que se muestren dinámico, procedimentales y significativo (SILVA \& ZANON, 2000).

De acuerdo con Borges (1997), los estudiantes no son desafiados a explorar, desarrollar y evaluar sus propias ideas y los planes de estudios de ciencias no se ofrecen oportunidades para el enfoque y propósitos de la ciencia y la investigación científica. Hoy en día se tiene en las actividades experimentales el objetivo de "promover las interacciones sociales que hagan que las explicaciones sean más accesibles y eficientes" (Gaspar, 2009, p. 24). Además de ayudar a promover la reflexión de los alumnos (Alvarado, 2009), Gaspar (2009) destaca que la actividad experimental tiene ventajas sobre la teórica, pero ambos deben caminar juntas, pues una es el complemento de la otra.

El autor enfatiza que el experimento en sí mismo no es capaz de desencadenar una relación con el conocimiento científico, sino en la combinación de la teoría con la práctica. El autor también resalta las ventajas de las clases prácticas, de demostración o experimentación.

Para Demo (2002, p. 90):

La capacidad de enfrentarse con cualquier tema es una construcción: se Condensa en la habilidad de saber reconstruir el conocimiento, hacer frente a cualquier desafío de conocimiento, porque sabe pensar, aprender a aprender, maneja de manera creativa lógica, el razonamiento, la argumentación, la deducción y la inducción, la teoría y la práctica. Esta capacidad de enfrentarse con cualquier tema pertinente, en el decir de Demo (2002, p. 91) es "[...] una instrumentación esencial de la competencia humana."

Perrenoud (2000, p. 29) explica que la mayor parte de los conocimientos científicos es contrario a la intuición, por lo tanto, es importante que los alumnos en las clases experimentales se confrontem con los límites de su propio conocimiento y desmoronarse de ideas intuitivas.

Sin embargo, lo que observamos en la práctica del ambiente escolar es una enseñanza de la Ciencia y de la Biología muy distante del alumno, lleno de información que en vez de ayudar en su desarrollo termina por no facilitar la formación de una red de conocimientos con sentido significativo y de fácil aplicabilidad en su vida cotidiana.

Este sentido o esta sensibilización se presenta a cada uno de forma diferente, de acuerdo con el grado de desarrollo y de entendimiento del individuo (LIMA, 1984). No es, por lo tanto, el estímulo 
que lleva al organismo a la actividad, como si el cuerpo fuera una caja vacía. Es preciso que el organismo esté sensibilizado para "darse cuenta" de los estímulos (LIMA, 1984).

\subsection{LA VISIÓN CONSTRUCTIVISTA EN LA ENSEÑANZA DE LAS CIENCIAS.}

Muchos de los modelos de enseñanza se basan en la teoría del desarrollo cognitivo de Jean Piaget, el cual establece que la mente humana a lo largo de su existencia va aumentando su organización interna y adaptándose al medio. Fontana y Cruz (1997) plantean que el desarrollo del conocimiento humano está inmerso en el estudio científico genético realizado por Piaget, dirigido principalmente a la cuestión de cómo el ser humano elabora sus conocimientos acerca de la realidad, llegando a construir, en el transcurso de su historia, sistemas científicos complejos y con un alto nivel de abstracción.

Nueva informaciónes provocan desequilibrios y reestructuraciones haciendo nuevos esquemas mentales, alcanzando un nuevo aprendizaje. Es evidente que la concepción piagetiana, se basa en la observación de experiencias vividas; los niños interactuando con los objetos que lleva al establecimiento de la organización y estructuración del proceso de formación del conocimiento por etapas, que culminó con la explicación de la realidad a partir de las experiencias previamente diseñadas.

Para Piaget el individuo al actuar sobre el medio al que pertenece, incorpora a sí elementos y a través de ese proceso de incorporación llamado por él, de asimilación, cosas y hechos del medio ganan significación para el individuo. Al mismo tiempo en que las nuevas ideas y conceptos son incorporados al sistema de relaciones, ideas y conceptos ya existentes son modificados por lo que ya ha sido asimilado. Este proceso de modificación que se opera en las estructuras de pensamiento del individuo es llamado por Piaget de alojamiento (FONTANA Y CRUZ, 1997).

Desde esta perspectiva, enseñar es provocar desequilibrios estructurales en los mapas mentales ya formateados, proporcionando condiciones para que el alumno alcance el equilibrio y se reestructure a nivel cognitivo. En este tiempo es importante destacar que el proceso de asimilación gradual provoca una transformación de los reflejos, que poco a poco se van diferenciando y se está haciendo cada vez más complejos y flexibles dejando de ser simples respuestas estereotipadas o etiqueten a estímulos previstos.

Este proceso da origen a esquemas de acción. Y es por medio de los esquemas de acción que el niño empieza a conocer la realidad, asimilándose a y atribuyéndole significados. Otro aspecto relevante a considerar, es que esta teoría debe estar asociada a acciones y demostraciones, así como oportunamente al trabajo práctico, el cual permite al estudiante experimentar la experimentación. Fontana y Cruz (1997), citando a Piaget ponen el cual permite al estudiante experimentar la 
experimentación. Fontana y Cruz (1997), citando a Piaget ponen que el sujeto tiene que actuar sobre los objetos; desplazarlo, agruparlos, combinarlos, separarlos y ajuntarlo.

Con el fin de que sus acciones se desplacen de su propio cuerpo a los objetos. De esta forma el niño paulatinamente va transfiriendo sus acciones y construyendo sus esquemas. Sus acciones ahora son repetidas debido a los efectos interesantes que producen y a los pocos, medios y fines se están diferenciados y las acciones comienzan a ganar intencionalidad.

Se puede inferir que Piaget no se molestó en estudiar la influencia del medio en la construcción del conocimiento, o sea, como el medio actúa en este proceso formativo de la cognición. Hoy se sabe que la integración de genotipo y medio interfiere directamente en el desarrollo del conocimiento de cada individuo tanto cognoscitivo como físico los que los genetistas llaman fenotipo.

Sin embargo, dentro del enfoque de esta Población, la dimensión socio-histórica de la psique, todo lo que es específicamente humano y distingue al hombre de otras especies se originan de su vida en sociedad, sus modos de percibir, representar, explicar y actuar sobre el medio, de sus sentimientos en relación al mundo, al otro y a sí mismo. En fin, su funcionamiento psicológico va constituyendo en sus relaciones sociales. Referente a esta interactividad con el medio, Fontana y la Cruz (1997), plantean que en este proceso interactivo, las reacciones naturales heredadas biológicamente, de respuestas a los estímulos del medio (tales como: la percepción, la memoria, las acciones reflejas, las reacciones automática si las asociaciones simple) se entrelazan los procesos culturalmente organizados y se van transformando en los modos de acción, de relación y de representación característicamente humanos. Parafraseando Vygotsky ellas destacan que, a diferencia de las otras especies, el hombre, por el trabajo, transforma el medio.

Por lo tanto, las actividades experimentales, encajan perfectamente en esta concepción ya que a través de la misma el alumno puede actuar directamente en el proceso de construcción de su conocimiento interactuando con los equipos presentes en el laboratorio, así como con los otros individuos. (MOREIRA, 1999). Por lo tanto, las actividades experimentales, encajan perfectamente en esta concepción ya que a través de la misma el alumno puede actuar directamente en el proceso de construcción de su conocimiento interactuando con los equipos presentes en el laboratorio, así como con los otros individuos. (MOREIRA, 1999).

Según Lima et al (1999), la experimentación proporciona la inter-relación entre la teoría y la práctica, lo que permite que el alumno sea el sujeto de la construcción de su propio conocimiento, lo que permite que el sujeto actúe, haciendo la conexión interpretativa entre los fenómenos y procesos naturales observados, basados en un modelo no sólo en el conocimiento 40 científico, pero a través de los saberes y las hipótesis planteadas por los estudiantes, frente a las situaciones/problema presentadas. 
Existe una fundamentación psicológica y pedagógica que defiende la necesidad de proporcionar al niño y al adolescente el ejercicio de habilidades en la perspectiva de la cooperación, la concentración, la organización, el manejo de equipos. Posibilitando que el alumno pueda experimentar el método científico, superando las etapas de la observación de fenómenos, del registro sistematizado de datos, la formulación y prueba de hipótesis y la inferencia de conclusiones (CAPELETO, 1992).

Moraes (1998) considera que la construcción de nuevos conocimientos debe sobresalir por el equipaje, en términos de conocimiento, que los alumnos traen en su historial. Aunque intuitivos y derivados, teniendo en cuenta que el proceso de aprendizaje pasa por la desestructuración y reformulación de los conocimientos a través del diálogo y la reflexión, ofreciendo juicios constructivistas, las cuales pueden implicar varias disciplinas al mismo tiempo, estableciendo acciones interdisciplinares y contextualizadas.

Las actividades experimentales pueden ser utilizadas como contrapunto a la teorización de las clases magistrales, convirtiéndose en un potente recurso pedagógico en el proceso de adquisición de nuevos conocimientos. La experimentación en la práctica tiende a facilitar la fijación de contenidos a ella relacionados, con efecto, descartando la idea de que las actividades experimentales deben servir únicamente para la ilustración de teorías (CAPELETTO, 1992).

Dentro de la perspectiva de la clase práctica cuya concepción ilustrativa se evidencia a través de la secuencia de los procedimientos realizados por el profesor, que después de la clase expositiva conduce a los alumnos en el laboratorio, de forma que estos alumnos puedan, por la actividad experimental dirigida, lleguen a sus propias conclusiones acerca de lo que se ha teorizado. De esta forma se evidencia que las actividades experimentales deben salir del nivel simplista, de un campo meramente técnico y garantizar una transmisión eficiente del conocimiento científico. (LIMA et al., 1999).

Sin embargo Fracalanzaet al, (1986), plantea que una postura experimental debe estar vinculada a la idea de la explotación del nuevo, la incertidumbre de alcanzar el éxito, la discusión de los resultados de la investigación, la acción y el contacto con el fenómeno estudiado. Según los parámetros curriculares nacionales (PCN, el simple hacer no significa necesariamente construir conocimientos y aprender ciencias (Brasil, 1998).

Sollé y Coll (2006) plantean que en la perspectiva constructivista el conjunto de postulados toma en cuenta el proceso compartido de enseñanza, en el cual el alumno con la intermediación del profesor puede construir el conocimiento de manera que sea competente, resolver situaciones/problema mediante el uso de los conceptos aprendidos en el proceso.

Por lo tanto, se puede constatar que la concepción constructivista de las actividades experimentales, asumidas en las clases prácticas, se caracteriza a través de la consideración del 
conocimiento previo del alumno, de forma que el mismo a través de la asimilación de nuevos conceptos reestructura nuevos mapas mentales a partir del conocimiento previo. La enseñanza, dentro de esta corriente, consiste en el financiamiento de actividades desafiantes que lleven al educando a buscar nuevos conocimientos [...] donde las estructuras cada vez más complejas, que van siendo construidas [...]" (CASTRO; CARVALHO, 2001, p. 183).

\subsection{LA INTERACCIÓN DE LA TEORÍA Y LA PRÁCTICA EN LA ENSEÑANZA DE LAS CIENCIAS: EL CONTEXTO DE LAS}

En las escuelas de enseñanza media, las ciencias de la naturaleza se encuentran separadas por disciplinas de las cuales implican la química, la biología y la física. La matemática y sus tecnologías constituyen un área distinta. El desarrollo del conocimiento científico básico en estas escuelas está condicionado al trabajo pedagógico realizado por los profesores, los cuales tienen la oportunidad de impartir los contenidos curriculares dentro de la perspectiva interdisciplinar, de forma transversal y contextualizada, de acuerdo con los PCN y las directrices contenidas en los mismos.

Hay temas cuyo estudio requiere un enfoque particularmente amplio y diversificado. Algunos de ellos fueron introducidos en los parámetros curriculares nacionales, que los denominados Temas Transversales y los caracteriza como temas que "tratan de procesos que están siendo intensamente vividos por la sociedad, las comunidades, las familias, los estudiantes y los educadores en su día a día. Son debatidos en diferentes espacios sociales, en la búsqueda de soluciones y alternativas, confrontando posicionamientos diversos, tanto en relación con la intervención en el ámbito social más amplio en cuanto a la actuación personal.

'En este contexto Macedo (1998, p. 23) plantea que "el conjunto de temas transversales que incluye la ética, la pluralidad cultural, medio ambiente, salud y orientación sexual. De estos, al menos, los tres últimos se relacionan más fácilmente con el área de ciencias..."

Teniendo en cuenta las diferentes concepciones relacionadas con el proceso de enseñanza y aprendizaje puestos en los PCN, se percibe que las competencias y habilidades a ser desarrolladas forman parte del contexto de cada disciplina y que la interdisciplinaridad y los temas transversales pueden ser trabajados en las diferentes disciplinas, de forma que las competencias y habilidades están inter-relacionadas entre las áreas. Según Morin (2001) el conocimiento de las informaciones aisladas es insuficiente, lo importante es que estas informaciones estén situadas dentro de un contexto.

La educación para la ciudadanía requiere, por tanto, que los problemas sociales sean presentadas para el aprendizaje y la reflexión de los alumnos. La inclusión de temas sociales en el currículo escolar no es una preocupación sin precedentes. Estas temáticas ya han sido discutidas e incorporadas a las áreas vinculadas a las Ciencias Sociales y las Ciencias Naturales, llegando incluso, 
en algunas propuestas, a constituir nuevas áreas, como en el caso de los temas de Medio Ambiente y Salud.

Los Parámetros Curriculares Nacionales incorporan esta tendencia y la incluyen en el plan de estudios de manera de componer un conjunto articulado y abierto a nuevos temas, buscando un tratamiento didáctico que contemple su complejidad y su dinámica, dándoles la misma importancia de las áreas convencionales.

El plan de estudios gana en flexibilidad y apertura, una vez que los temas pueden ser priorizados y contextualizados de acuerdo con las diferentes realidades locales y regionales, y otros temas que pueden ser incluidos (BRASIL, 1999, p. 25).

Las ciencias de la naturaleza y sus tecnologías evidencian a través de la contribución al desarrollo científico y tecnológico. De esta forma subtiende que las competencias y habilidades relacionadas con ellas deban proporcionar la formación de los individuos, no sólo competentes técnicamente, pero también críticos, reflexivos y conscientes de sus derechos y deberes como ciudadanos insertos en una sociedad y co-responsable por el planeta en el que habita. Por esta razón se considera que el profesor de ciencias necesita apropiarse de estos importantes conceptos con el fin de proponer y desarrollar estrategias pedagógicas que posibiliten la preparación del alumno ante el mundo científico y tecnológico.

Hasta hace poco tiempo, la gran cuestión escolar era el aprendizaje - exclusiva o preferencial - de conceptos. Estábamos dominados por la idea de que conocer es acumular conceptos; ser inteligente implica articular lógicamente grandes ideas, estar informado sobre grandes conocimientos ...

Este tipo de clase, insisto, sigue teniendo lugar, pero cada vez más se hace necesario también el dominio de un contenido llamado procedimental", o sea de la orden del saber como hacer. Vivimos en una sociedad cada vez más tecnológica, en la que el problema no siempre está en la falta de información, ya que el equipo tiene cada vez más el poder de procesarlas, guardarlas o actualizarla. La cuestión está en encontrar, interpretar esta información, en la búsqueda en la búsqueda de la solución de nuestros problemas (MACEDO 1999. p. 8).

Dentro de la concepción conceptual de competencias y habilidades, Machado (2000 apud Cruz, 2005) plantea que el concepto de competencia está vinculada a la capacidad de transformar la tecnología en el producto. Este concepto se encuentra muy distante de la concepción educativa, sin embargo dentro del pensamiento técnico y de mercado. El INEP, busca conceptualizar las competencias y habilidades de manera continua y conectada, partiendo del principio conceptual de la estructuración de la inteligencia anunciado por Piaget, asociado a los conceptos de Población, de forma que estas modalidades estructurales cognitivas que permitan a las acciones y operaciones para establecer relaciones con y entre los objetos, situaciones, fenómenos y personas. 
Las competencias son las modalidades estructurales de la inteligencia, o mejor, las acciones y las operaciones que utilizamos para establecer relaciones con y entre los objetos, situaciones, fenómenos y personas que deseamos conocer. Las habilidades se derivan de las competencias adquiridas y se refieren al plan inmediato del "saber hacer". Por medio de las acciones y operaciones, las habilidades perfeccionan y se articulan, posibilitando nueva reorganización de las competencias (INEP, 1999, p.7).

La competencia es la capacidad que las personas desarrollan de articular, vincular los diferentes saberes, conocimientos, actitudes y valores, construidos por medio de su vivencia y por medio de los conocimientos construidos en la escuela. Esa articulación y la relación se construyen a partir de las necesidades de la vida diaria, de las emociones y del enfrentamiento de las situaciones difíciles con las que tenemos que dialogar (RAMOS, 2001, apud CRUZ, 2005, p. 29).

Entende-se que habilidades são recursos cognitivos inatos, ou adquiridos de forma instrucional, que capacitam o indivíduo a resolver situações/problemas. Com efeito, o indivíduo habilidoso, ou seja, hábil em ação particular ou generalizada é aquele que detém o conhecimento sobre como fazer, e o fazer propriamente dito. O indivíduo ao nascer traz consigo alguns poucos esquemas, de forma que a partir desta herança, constrói outros esquemas de maneira contínua.

Perrenoud (1999), citando al sociólogo Bourdieu, pone que el conjunto de esquemas constituidos en un momento dado de la vida se llama el habitus, el cual es definido por el sociólogo como "un pequeño lote de esquemas que permiten generar una infinidad de prácticas adaptadas a situaciones siempre renovadas, sin jamás se constituya en principios explícitos" (BOURDIEU, 1972 apud PERRENOUD. 1999).

Según el INEP, las habilidades están ligadas a la acción inmediata del "saber-hacer", de manera que las acciones y operaciones realizadas oportunizem el perfeccionamiento de las habilidades, que de manera articulada nueva reorganización de competencias (BRASIL, 1999).

Hengemühle (2010) plantea que en la actualidad el individuo es invitado a hacer ejercicio, al máximo, el cerebro de manera que el ser humano se convierte en un individuo capaz de comprender la vida y resolver situaciones problemas de su tiempo, fundamentado en conocimientos y valores que lleven al sujeto a un actuar competente.

Competencias son herramientas que el individuo desarrolla, apropiarse de conocimientos que habiliten para el ejercicio de las funciones requeridas por el medio en el cual está insertado.

El conocimiento es entendido como lo que muchos denominan simplemente saber. La habilidad se refiere al saber hacer relacionado con la práctica del trabajo, trascendiendo la mera acción motora. El valor se expresa en el saber ser, en la actitud relacionada con el juicio de la pertinencia de la acción, 
con la calidad del trabajo, la ética del comportamiento, la convivencia participativa y solidaria y otros atributos humanos, como la iniciativa y la creatividad...(MEC/SETEC, 2005 -6 $6^{\text {a }}$ edición).

En este contexto se puede inferir que las habilidades abarcan aspectos relacionados con el cognitivo, el cual a través de la formación de esquemas mentales alcanza sucesivas equilibrações, proporcionando al individuo el desarrollo de competencias que posibilitan el ejercicio de determinadas funciones (PERRENOUD, 1999, p. 27).

Considerando las competencias y habilidades, se resalta el papel del profesor en la mediación del desarrollo de las mismas. Con efecto, se entiende importante en esa interfase, los recursos pedagógicos que pueden ser utilizados con vistas al fin deseado.

A fin de contribuir con el profesor, los PCN proponen los ejes cognitivos guía de cada asignatura de la educación básica, proporcionando que este profesor actúe pedagógicamente y de forma dialogada, trabajando las competencias y habilidades. Como ejemplo usa los ejes cognitivos con sus respectivas competencias y habilidades de la asignatura de ciencias.

\subsection{EL CURRÍCULO EN LA FORMACIÓN DE PROFESORES: EL ENFOQUE DE LA PRÁCTICA DE LAS DISCIPLINAS DE LA GRADUACIÓN.}

Dentro del contexto de la enseñanza de ciencias y biología, se deben resaltar las dificultades que el profesor se enfrenta desde la promulgación de la LDB - 9394/96, culminante con la construcción de los parámetros curriculares nacionales (PCN. El currículo de las disciplinas sufrió un realineamiento, proporcionando cambio de tamaño de los contenidos, los cuales orientados por las directrices curriculares nacionales fueron agrupados y nombrados por matrices curriculares.

Incluso impulsados por los objetivos de las competencias y habilidades, la biología, así como otras disciplinas del área de las ciencias de la naturaleza, siguen etiquetadas como disciplinas críticas ante el proceso de enseñanza y aprendizaje. Sin embargo, "No se puede traducir esta problemática como una cuestión no sólo del profesor, en primer lugar, porque él también es víctima del sistema [...]" (DEMO, 2007, p. 42).

La problemática de la enseñanza y aprendizaje de las ciencias, más específicamente en biología, involucra diversos factores, entre ellos destaca la formación inicial del profesor, así como la deficiencia en los programas de formación continua ofrecidos por una variedad de instituciones públicas y privadas que no priman por programas más específicos.

A menudo, muchos educadores ha advertido de que la enseñanza de las ciencias pasa por dificultades, incluso señalando para ello algunas de las causas: deficiente preparación profesional del profesor; la falta de oportunidad y los medios para el profesor de actualización; deficiencias en las condiciones materiales de la mayoría de las escuelas (Hennig, 1998, p. 14). La difusión de los cursos 
de licenciatura, incluyendo la disminución del tiempo de finalización para los llamados cursos de licenciatura específica, con el fin de suplir la carencia de docentes con nivel superior tiene acarretado caída en la calidad de la formación de estos docentes. Principalmente en el área de las ciencias de la naturaleza, ya que algunas instituciones de educación superior no cuentan con condiciones de infraestructura que supram las necesidades de las lecciones sobre la práctica laboratoriales.

Aunque la ley 9394/96 ha sido creada con el fin de orientar la formación de profesores en Brasil, los cursos de licenciatura, cuyo objetivo es mejorar la calidad de la formación de estos profesionales están por debajo de lo esperado. Por lo tanto se infiere que se hacen necesarios cursos de licenciatura que oportunizem la construcción de saberes facilitadores del ejercicio de la docencia, lo que permite a estos profesionales, cualificación para desarrollar la enseñanza de las ciencias en la educación básica. (PACHECO, 2012).

De hecho, existe un grave peligro de que profundas transformaciones asociadas a las orientaciones constructivistas hoy emergentes (Gil-Pérez, 1991a) queden desvirtuadas en su aplicación concreta: no basta con estructurar cuidadosamente y profundamente un plan de estudios si el profesor no recibir una preparación adecuada para aplicarlo (GIL-PÉREZ y CARVALHO, 2006, p. 9).

Sin embargo, el problema no se resuelve sólo ofreciendo a los profesores instrucciones más detalladas, a través de manuales o cursos ad hoc: se hace necesaria una profunda revisión de la formación - inicial y permanente - de los profesores, extendiendo la misma a las adquisiciones de las investigaciones sobre el aprendizaje de las ciencias y, en especial, a las propuestas de la orientación constructivista (BRISCOE, 1991, apud GIL-PÉREZ y CARVALHO, 2006, p. 10).

Cursos de licenciatura y de baja calidad, diseminados por todo el territorio nacional, ha revelado grandes perjuicios en la formación inicial y permanente de los docentes del área de las ciencias de la naturaleza. Con relación a este aspecto se infiere que una de las principales dificultades que enfrentan es con respecto a la discapacidad en el ámbito del contenido de la asignatura impartida, tanto en el ámbito del conocimiento teórico como en el práctico experimental. En efecto, este hecho provoca el descrédito del profesional ante los alumnos, resultando en la mayoría de las veces indisciplina y falta de respeto para el profesional y la escuela.

Es cierto que el ejercicio de la profesión docente requiere el dominio de saberes que fundamentan el proceso de enseñanza-aprendizaje. Fiorentini et al (1998, p. 312), define a conocer como "[...] un modo de conocer/saber más dinámico, menos sistematizado y más articulado a otras formas de saber y hacer, relativos a la práctica no tienen estrictas normas formales de validación."

Es cierto que el ejercicio de la profesión docente requiere el dominio de saberes que fundamentan el proceso de enseñanza-aprendizaje. Fiorentini et al (1998, p. 312), define a conocer 
como "[...] un modo de conocer/saber más dinámico, menos sistematizado y más articulado a otras formas de saber y hacer, relativos a la práctica no tienen estrictas normas formales de validación."

Aunque los cursos de formación inicial y permanente hayan evolucionado, aún no han alcanzado la madurez en cuanto a la preparación de los docentes en lo que se refiere al conocimiento científico innovador y dentro de las perspectivas constructivistas, de forma que aún predominan instituciones cerradas en sí mismo, ora por un academicismo exacerbada o un empirismo tradicional, enyesado en currículum tecnicista sin ninguna concepción progresista (NÓVOA,1992).

De cierta forma, el profesor del área de ciencias considera al alumno como un simple receptor de conceptos previamente elaborados, a pesar de que las nuevas concepciones pedagógicas, para demostrar que la dinamicidad de las clases teórico/magistrales, de la contextualización de contenidos y la valoración del conocimiento previo del alumno, proporcionen mejores resultados en el aprendizaje.

Sin embargo, de acuerdo con Tardif (2002, p. 39), "[...] el profesor ideal es alguien que debe conocer su materia, su disciplina y su programa, además de poseer ciertos conocimientos relativos a las ciencias de la educación y la pedagogía y el desarrollo de un saber práctico basado en su experiencia cotidiana con los alumnos".

\section{CONSIDERACIONES FINALES}

Por lo tanto la Enseñanza de las Ciencias es muy importante, ya que aborda los principios científicos generales y, también, aplicaciones tecnológicas. Los conceptos y teorías científicas no tienen valores en sí mismos, como sistemas abstractos de pensamiento, sino como instrumentos que nos ayudan a entender el mundo en el que vivimos con el fin de orientar nuestras acciones a nivel individual y social.

Es en la época de la escuela que los estudiantes aprenden a gustar o no de la materia de Ciencias, ya que la misma posibilita la comprensión de los acontecimientos pasados, o sea, permite entender los procesos que rigen nuestra vida en la Tierra. Es posible comprobar la expectativa de los educandos durante la semana para tener clases de Ciencias, porque siempre le preguntan lo que será el pasado de diferente en el aula.

A través de toda la dificultad de los alumnos frente a esta materia fue posible informar sobre la importancia de las clases prácticas experimentales para enseñar Ciencias en la educación básica. Después de mucha investigación, se puede entender que la experimentación durante las clases, no sólo está en despertar el interés por la Ciencia en los alumnos, sino de hacer más comprensible los contenidos abordados en la teoría.

La práctica vinculada a la teoría hace mucha diferencia para una clase contextualizada, donde los estudiantes pueden ver la importancia de los contenidos abordados en la enseñanza de las Ciencias, 
además de ofrecer a los mismos una mayor claridad para que puedan realmente interpretar o sea hacer parte del estudio en cuestión.

Es visible hoy en día la necesidad de la experimentación durante las clases como instrumento de enseñanza, porque el estímulo y el interés de los estudiantes pasa a ser mucho mayor, ya que los mismos pueden visualizar el contenido de forma diferente, o sea, pasan a analizar ciertas cuestiones como si hicieran parte de ella.

Por lo tanto, la práctica experimental tiene un papel más amplio de lo que se espera, porque desarrolla en los alumnos un mayor interés, además de despertar habilidades que no aparecía en las clases teóricas, por ejemplo. 


\section{FUENTES BIBLIOGRÁFICA}

ALVES FILHO, J. P. Regras da Transposição Didática Aplicadas ao Laboratório Didático. In: II Encontro Nacional de Pesquisa em Educação em Ciências (ENPEC), 1999, Valinhos, SP, Anais.

AZEVEDO, Maria Cristina P. Stella de. Ensino por investigação: problematizando as atividades em sala de aula. In: CARVALHO, Anna Maria Pessoa de (Org.). Ensino de ciências: unindo a pesquisa e a prática. São Paulo: Cengage Learning, 2009.

BAZIN, M. (1987). Threeyearsof living science in Rio de Janeiro: learningfromexperience. ScientificLiteracyPapers, 67-74. Brasil. (1998). Secretaria de Educação Fundamental. Parâmetros curriculares nacionais:Ciências Naturais/Secretaria de Educação Fundamental. Brasília: MEC/SEF.

BIZZO, Nélio. Ciências: fácil ou difícil? - 2ª ed. São Paulo: Editora Ática, 2007. p.24 75.

BORGES, A.T. O papel do laboratório no ensino de ciências. In: MOREIRA, M.A.; ZYLBERSZTA J.N.A.; DELIZOICOV, D.; ANGOTTI, J.A.P. Atlas do I Encontro Nacional de Pesquisa em Ensino de Ciências. Editora da Universidade - UFRGS, Porto Alegre, RS, 1997. 2-11.

BRASIL, Ministério da Educação, Secretaria de Educação Média e Tecnológica. Parâmetros Curriculares Nacionais: Ensino Médio. Brasília: Ministério da Educação, 1999. 360 p.

BRASIL, Ministério da Educação. Parâmetros Curriculares Nacionais Ensino Médio. Parte I - Bases Legais. 2000b. Disponível em: http://portal.mec.gov.brarquivos/pdf/blegais.pdf. Acesso em 20 de set. 2012.

CAPELETTO, A. Biologia e Educação ambiental: Roteiros de trabalho. Editora Ática, 1992. p. 224.

CARNEIRO, Henrique S.. História da Ciência, da Técnica e do Trabalho no Brasil. Disponível em: <http://nuevomundo.revues.org/573>. Acesso em: 16 maio 2011. 89

CARVAlHO, Anna Maria Pessoa de et al. Ciências no Ensino Fundamental: o conhecimento físico. São Paulo: Scipione, 2007. 37

DEMO, Pedro. Os desafios modernos da educação. 14ª ed. Petrópolis: Vozes, 2007.

FIORENTINI, D.; SOUZA JÚNIOR, A. J. de.; MELO, G. F. A. de. Saberes docentes: um desafio para acadêmicos e práticos. In: GERADI, C. M. G.; FIORENTINI, D.\& PEREIRA, E. M. de A. (Orgs.). Cartografia do trabalho docente: professor (a)-pesquisador(a). Campinas, SP: Mercado de Letras: Associação de Leitura do Brasil - ALB, 1998.

FONTANA, Roseli Aparecida Cação; CRUZ, Maria Nazaré da. A abordagem Piagetiana. In: FONTANA, Roseli Aparecida Cação; CRUZ, Maria Nazaré da.Psicologia e trabalho pedagógico. São Paulo: Atual, 1997. Cap. 4, p. 43-67. 90

FRACALANZA, H. et al. O Ensino de Ciências no $1^{\circ}$ grau. São Paulo: Atual. 1986. p.124.

FREIRE, P. Pedagogia da Autonomia. Rio de Janeiro: Paz e Terra, 1997.

GALIAZZI, M. C.; ROCHA, J. M .B; SCHMITZ, L. C.; SOUZA, M. L.; GIESTA, S. e GONÇALVES, F. P. Objetivos das atividades experimentais no ensino médio: a pesquisa coletiva como modo de formação de professores de ciências. Ciência e Educação, v. 7, n. 2, p. 249263, 2001.

GARCIA, Lenise Aparecida Martins. Transversalidade e Interdisciplinaridade. Disponível em: <http://4pilares.net/text-cont/garcia-transversalidade.htm>. Acesso em: 08 ago. 2011.

GARRETT, R. M. y ROBERTS, I.F., 1982. Demonstration Vs. smallgrouppracticalwork in Science Education: a criticalreviewofstudiessince 1900, Stzldies in Science Education., 9, PP. 109-146 
GASPAR, Alberto. Experiências de Ciências para o Ensino Fundamental. São Paulo: Ática, 2009.

GASPARETTO JUNIOR, Antonio. Revolução Científica. Disponível em: <http://www.infoescola.com/historia/revolucao-cientifica/>. Acesso em: 04 mar. 2011.

GIL-PÉREZ, Daniel. Formação de professores de ciências: tendências e inovações/ Daniel Gil-Pérez, Anna Maria Pessoa de Carvalho; revisão técnica da autora; [tradução Sandra Valenzuela]. $8^{a}$. Ed. - São Paulo: Cortez, 2006. - (Coleção Questões da Nossa Época: v. 26).

GIORDAN, M. O papel da experimentação no ensino de ciências. Química Nova na Escola, n. 10,p. 43-49, 1999. if.ufmt.br/eenci/artigos/Artigo_ID174/v7_n1_a2012.pdf. Acesso em 24/11/2013.

HENGEMÜHLE, Adelar. Desenvolver Habilidades, Formar Para As Competências: Modelos Novos, Práticas Antigas. Osório: Mímeo, 2010.

HODSON, D. Hacia um enfoque más crítico deltrabajo de laboratório. Enseñanza delas Ciencias, v.12, n. 13, p.299-313, 1994. http://portaldoprofessor.mec.gov.br/fichaTecnicaAula.html?aula=554. Acesso em 23/07/2014. http://www.ucs.br/etc/conferencias/index.php/anpedsul/9anpedsul/paper/viewFile/2782/286. Acesso em 24/11/2013. http://www.maxwell.lambda.ele.puc-rio.br/11290/11290_4.PDF. Acesso em 24/11/2013. http://if.ufmt.br/eenci/artigos/Artigo_ID174/v7_n1_a2012.pdf. Acesso em 24/11/2013.

HOFFMANN, Jussara. Avaliação mediadora: uma prática em construção da préescola à universidade. Porto Alegre: Mediação, 2003 - 20 a edição.

KRASILCHIK, Myriam. REFORMAS E REALIDADE o caso do ensino das ciências. São Paulo em Perspectiva, São Paulo, v. 14, n. 1, p.85-93, mar. 2000.

LIMA, K. E. C. de; VASCONCELOS, S. D. Análise da metodologia de ensino de ciências nas escolas da rede municipal de Recife. Ensaio: Avaliação e Políticas Públicas em Educação vol. 14 nº 52 Rio de Janeiro jul/set. 2006.

LUNETTA, V. \& HOFSTEIN, A. (1991). Simulationandlaboratorypracticalactivity. In B. Woolnough (Ed.), Practicalscience (pp 125-137).

LUNETTA, V. N. Atividades práticas no ensino da Ciência. Revista Portuguesa de Educação, v. 2, n. 1, p. 81-90, 1991.

MACEDO, L. (1999). Competências e habilidades: elementos para uma reflexão pedagógica. Brasília: INEP.

MORAES, R. O significado da experimentação numa abordagem construtivista: O caso do ensino de ciências. In: BORGES, R. M. R.; MORAES, R. (Org.) Educação em Ciências nas séries iniciais. Porto Alegre: Sagra Luzzato. 1998. p. 29-45.

MOREIRA, Marco Antonio. PESQUISA BÁSICA EM EDUCAÇÃO EM CIÊNCIAS: UMA VISÃO PESSOAL. Revista Chilena de Educación Científica, Chile, n. , p.1-12, 17 out. 2004.

MATOS, Maria Margarida O. M. F. Trabalho experimental na aula de Ciências Físico-Químicas do $3^{\circ}$ Ciclo do Ensino Básico: Teorias e práticas de professores. 2001. Tese (mestrado em Educação) - Faculdade de Ciências, Universidade de Lisboa, Portugal.

MORIN, Edgar. Os sete saberes necessários à educação do futuro. Brasília: UNESCO, 2001.

NÓVOA, A. (org). O professor e sua formação. Lisboa: D. Quixote, 1992, Temas de educação.

PAIVA, Fernando Martins de. Seara da Ciência: contribuições à formação docente de licenciados de física/Fernando Martins de Paiva. Dissertação (mestrado) - Universidade Federal do Ceará, Centro de Ciências, Mestrado Profissional em Ensino de Ciências e Matemática - ENCIMA. Fortaleza - 2012. 134 p. 92 
PERRENOUD, P. Construir as competências desde a escola. Porto Alegre: Atemed, 1999.

PIAGET, Jean. Para onde vai a educação? Tradução de Ivette Braga, $14^{a}$ ed. Rio de Janeiro: José Olympio, 1998.

SILVA, Karolina Martins Almeida E. ABORDAGEM CTS NO ENSINO MÉDIO: UM ESTUDO DE CASO DA PRÁTICA PEDAGógICA DE PROFESSORES DE BIOLOGIA. 2010. 160 f. Dissertação (Pósgraduação Stricto Sensu) - Curso de Mestrado em Educação em Ciências e Matemática, Universidade Federal De Goiás, Goiânia - Go, 2010.

SILVA, L.H.de A.; ZANON, L.B. A experimentação no ensino de Ciências. In: SCHNETZLER, R.P.; ARAGÃO, R.M.R. Ensino de Ciências: Fundamentos e Abordagens. Piracicaba: CAPES/UNIMEP, 2000. $182 \mathrm{p}$.

SMITH, K.A. Experimentação nas Aulas de Ciências. In: CARVALHO, A.M.P.; VANNUCCHI, A.I.; SOLÉ, I. Disponibilidade para Aprendizagem. IN: COLL, C; MARTIN, E; MAURI, T; MIRAS, M; ONRUBIA, J; SOLÉ, I; ZABALA, A. O construtivismo na sala de aula. São Paulo: Editora Ática 2006, p. 29 -56 .

TAMIR, P. How are thelaboratoriesused ?JournalofResearch in Science Teaching, v. 14, n. 4, p. 311-316, 1977.

TARDIF, M.. Saberes docentes e formação profissional. 6. ed. Petrópolis: Vozes, 2002.

SEVERINO, Antônio Joaquim. Metodologia do Trabalho Científico. 23ª ed. São Paulo: Cortez, 2008.

SÉRÉ, M. G.; COELHO, S. M.; NUNES, A. D. O papel da experimentação no ensino da Física. Caderno Brasileiro Ensino de Física, v. 20, n. 1, p. 30-42, abr. 2003. Disponível em: http://stat.contabilidade.periodicos.ufsc.br. Acesso: 18/03/2010.

THOMAZ, M. F. A experimentação e a formação de professores de ciências: uma reflexão. Caderno Brasileiro de Ensino de Física, v. 17, n. 3, p. 360-369, dez. 2000. Disponível em: http://search.sweetim.com/search. Acesso em: 21/05/2010. 